\title{
ON DECOMPOSITION INTO STOCHASTICALLY INDEPENDENT COMPONENTS
}

\author{
S. P. LLOYD
}

1. Introduction. Let $\mathcal{G}$ be an arbitrary class of measurable sets in the probability space $(\Omega, \mathcal{F}, P)$. We denote by $\mathcal{G}^{s}$ the class of sets which are stochastically independent of $\mathcal{G}$; that is,

$$
\mathcal{G}^{s}=\{H \in \mathcal{F}: P(H \cap G)=P(H) P(G) \text { for all } G \in \mathcal{G}\} .
$$

If $\mathcal{G} \vee \mathcal{G}^{s}$ denotes the smallest $\sigma$-field containing $\mathcal{G}$ and $\mathcal{G}^{s}$, we say that decomposition (of $\mathcal{F}$ relative to $\mathcal{G}$ ) holds if $\mathcal{G} \vee \mathcal{G}^{8}=\mathfrak{F}$. In other words, when decomposition holds, every statistical experiment in the class under consideration (every $\mathfrak{F}$ set) can be expressed as a combination of (is in the $\sigma$-field generated by) experiments from the designated sublcass (sets in $\mathcal{G}$ ) and experiments independent of the subclass (sets in $\mathcal{G}^{s}$ ). Examples will be given where decomposition holds and where it fails, and a sufficient condition for decomposition to hold will be established.

2. Some properties of independence. We digress briefly to exhibit some elementary properties of the ${ }^{\prime}$ operation. We denote by $\theta$ the $\sigma$-field consisting of sets of probability 0 and their complements. The following relations hold for arbitrary subclasses $\mathcal{G}, \mathfrak{F}, \cdots$, of $\mathcal{F}$.

(i) $\mathcal{G}^{8}$ is closed under the formation of complements, proper differences, and (countable) disjoint unions (but not necessarily intersections),

(ii) $\mathcal{O}^{s}=\mathcal{F}, \mathfrak{F}^{s}=\mathcal{O}$,

(iii) $\mathcal{G}^{8} \supset \mathcal{O}$,

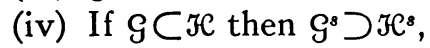

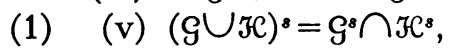

(vi) $\left(\mathcal{S} \cap \mathcal{H C}^{\mathrm{s}} \supset \mathcal{G}^{\mathrm{S}} \cup \mathcal{H}^{s}\right.$,

(vii) $\mathcal{G} C \mathcal{G}^{s s}\left(=\left(\mathcal{G}^{s}\right)^{s}\right)$,

(viii) $g^{s s s}=g^{s}$,

(ix) $g \cap g^{8} \subset \theta$,

(x) $\left(\mathcal{G} \cup \mathcal{G}^{s}\right)^{s}=\mathcal{O},\left(\mathcal{G} \cup \mathcal{G}^{s}\right)^{s s}=\mathcal{F}$

(xi) $\left(\mathcal{G} \vee \mathcal{G}^{s}\right)^{s}=\mathcal{O},\left(\mathcal{G} \bigvee \mathcal{G}^{s}\right)^{s s}=\mathcal{F}$

Proofs of the above properties are simple and straightforward, and are omitted. It is to be noted that the problem of when $\mathcal{G} \vee \mathcal{G}^{s}=\mathcal{F}$ holds is subsumed by the problem of when $\mathfrak{H}^{8 s}=\mathfrak{H}$ holds, from (xi).

Presented to the Society January 22, 1962 under the title On decomposition into independent components; received by the editors February 2, 1962. 
3. Conditional cumulative distributions. In all that follows we assume that $\mathcal{G}$ is a sub- $\sigma$-field of $\mathcal{F}$.

THEOREM 1. $H \in \mathcal{G}^{s}$ if and only if $P(H \mid \mathcal{G})=P(H)$ with probability 1.

Proof. $P(H \mid \mathcal{G})$ is the unique element of $L_{\infty}(\Omega, \mathcal{G}, P)$ satisfying

$$
P(H \cap G)=\int_{G} P(H \mid \mathcal{G})_{\omega} d P(\omega) \quad \text { for all } G \in \mathcal{G} \text {. }
$$

When $H \in g^{s}$ the left-hand side is equal to $P(H) P(G)$, and a solution of (2) is clearly $P(H \mid \mathcal{G})=$ const $=P(H)$ with probability 1 . Conversely, if $P(H \mid G)$ is constant with probability 1 then $P(H \cap G)$ $=P(H) P(G)$ holds for every $G \in \mathcal{G}$, whence $H \in \mathcal{G}^{8}$.

If $x$ is a real valued random variable we denote by $[x]$ the smallest $\sigma$-field with respect to which $x$ is Borel measurable, that is, $[x]$ is the class of sets $\{\omega: x(\omega) \in B\}$ for all linear Borel sets $B$. The conditional cumulative distribution of $x$ relative to $G$ will be denoted by

$$
F(\lambda, \omega)=P(x \leqq \lambda \mid g)_{\omega}, \quad-\infty<\lambda<\infty, \omega \in \Omega .
$$

We can and do assume that the conditional probability on the righthand side is determined for each $\lambda$ in such a way that $F(\cdot, \omega)$ is a bona fide cumulative distribution function for each $\omega \in \Omega[1$, p. 29].

Theorem 2. Suppose $F(\lambda, \omega),-\infty<\lambda<\infty, \omega \in \Omega$, is continuous on $-\infty<\lambda<\infty$ for each $\omega$ in the complement of a set of probability 0 . Then the random variable $\xi$ defined by

$$
\xi(\omega)=F(x(\omega), \omega)
$$

has the properties

(i) $[\xi] \subset[x] \vee \mathcal{G}$,

(ii) $[x] \subset[\xi] \vee \mathcal{G}$,

(iii) $[\xi] \subset \mathcal{G}^{8}$.

REMARK. P. Lévy is the first to use the independence property of random conditional cumulatives; Lévy gives no proof [2]. Since there seems to be no published proof, and since proof is not as simple as would seem off hand, we give it here.

We need first a measure theoretic lemma. Let $R$ denote the $\sigma$-field of linear Borel sets, and let $(\Omega \times \Lambda, \mathcal{G} \times R)$ denote the product measurable space of $(\Omega, \mathcal{G})$ with $(\Lambda, \Omega)$, where $\Lambda$ is the real line. If $\Gamma \subset \Omega \times \Lambda$ then $\Gamma_{\omega}$ denotes the $\omega$ section $\Gamma_{\omega}=\{\lambda \in \Lambda:(\omega, \lambda) \in \Gamma\}$. For any Borel set $B$, define

$$
F(B, \omega)=\int_{B} F(d \lambda, \omega)
$$


LEMma 1. If $\Gamma \in \mathcal{G} \times$ R then

$$
P\left(x(\omega) \in \Gamma_{\omega}\right)=\int F\left(\Gamma_{\omega}, \omega\right) d P(\omega), \quad \Gamma \in \mathcal{S} \times R .
$$

Proof. The properties of $F(\cdot, \cdot)$ insure that $\Delta_{1}(\Gamma)=\int F\left(\Gamma_{\omega}, \omega\right) d P(\omega)$

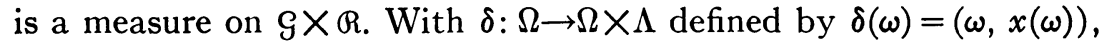
let $\Delta_{2}$ be the measure $\Delta_{2}(\Gamma)=P\left(\delta^{-1}(\Gamma)\right), \delta^{-1}(\Gamma) \in \mathcal{F}$, noting that $\Delta_{2}(\Gamma)$

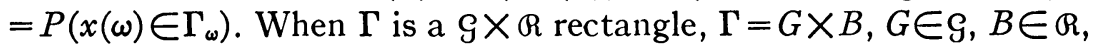
we have

$$
\begin{aligned}
\Delta_{2}(\Gamma) & =P(G \cap\{x \in B\}) \\
& =\int_{G} P(x \in B \mid \mathcal{G})_{\omega} d P(\omega) \\
& =\int F\left(\Gamma_{\omega}, \omega\right) d P(\omega)=\Delta_{1}(\Gamma)
\end{aligned}
$$

Since measures $\Delta_{1}$ and $\Delta_{2}$ coincide on $\mathcal{S} \times \mathcal{R}$ rectangles, they coincide on $\mathcal{G} \times R$, proving the lemma.

Proof of Theorem 2. Define the pseudoinverses of $F(\cdot, \omega)$ as

$$
\begin{aligned}
\bar{G}(u, \omega)= & \sup \lambda: F(\lambda, \omega) \leqq u \\
\underline{G}(u, \omega)= & \min \lambda: F(\lambda, \omega) \geqq u, \\
& 0<u<1, \omega \in \Omega .
\end{aligned}
$$

We have, for all values of the arguments,

$$
\begin{gathered}
F(\lambda, \omega)<u \quad \text { if and only if } \lambda<\underline{G(u, \omega),} \\
0<u<1, \quad-\infty<\lambda<\infty, \quad \omega \in \Omega .
\end{gathered}
$$

The assumed continuity of $F(\cdot, \omega)$ gives

$$
F(\underline{G}(u, \omega), \omega)=F(\bar{G}(u, \omega), \omega)=u, \quad 0<u<1,
$$

with probability 1 .

We will show that the conditional distribution of $\xi$ given $\mathcal{G}$ is the uniform-on-the-unit-interval distribution with probability 1 . For each $G \in \mathcal{G}$ and each $u, 0<u<1$, we have

$$
\begin{aligned}
& P(G \cap\{\xi<u\})=P(G \cap\{F(x(\omega), \omega)<u\}) \\
& =P(G \cap\{x(\omega)<\underline{G}(u, \omega)\}) \\
& =P\left(x(\omega) \in \Gamma_{\omega}\right)
\end{aligned}
$$

where $\Gamma \subset \Omega \times \Lambda$ is the set 


$$
\begin{aligned}
& \Gamma=(G \times \Lambda) \cap \Delta, \\
& \Delta=\{(\omega, \lambda): \lambda<\underline{G(u, \omega)\} .}
\end{aligned}
$$

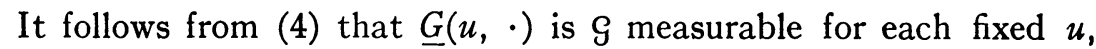
$0<u<1$; to see that $\Delta$ is $\mathcal{G} \times R$ measurable, observe that $\Delta=\lim _{n} \Delta_{n}$, with

$$
\begin{aligned}
\Delta_{n} & =\bigcup_{j=-\infty}^{\infty} D_{n, j} \times\left(-\infty, j / 2^{n}\right) \\
D_{n, j} & =\left\{\omega: j / 2^{n}<\underline{G}(u, \omega) \leqq(j+1) / 2^{n}\right\},
\end{aligned}
$$

and the approximating sequence is manifestly $\mathcal{G} \times \mathbb{R}$ measurable. Applying Lemma 1, we have, for each $G \in \mathcal{S}$ and each $u, 0<u<1$,

$$
\begin{aligned}
\int_{G} P(\xi<u \mid \mathcal{S})_{\omega} d P(\omega) & =P(G \cap\{\xi<u\})=P\left(x(\omega) \in \Gamma_{\omega}\right) \\
& =\int F\left(\Gamma_{\omega}, \omega\right) d P(\omega) \\
& =\int_{G} F(G(u, \omega)-0, \omega) d P(\omega) \\
& =u P(G), \quad G \in \mathcal{G}, 0<u<1,
\end{aligned}
$$

whence $P(\xi<u \mid \mathcal{G})=u$ with probability 1 , and this for each $u$, $0<u<1$.

That $\xi$ is $[x] \vee \mathcal{g}$ measurable follows easily from (4). It remains to show that $x$ is $[\xi] \vee \mathcal{g}$ measurable. Define random variables $\underline{x}$ and $\bar{x}$ by

$$
\begin{aligned}
& x(\omega)=\underline{G}(\xi(\omega), \omega) \\
& \bar{x}(\omega)=\bar{G}(\xi(\omega), \omega),
\end{aligned}
$$

(where $\omega \in \Omega$ ),

observing that $\underline{x}$ is $[\xi] \vee \mathcal{G}$ measurable, from (4), and that $\underline{x}(\omega) \leqq x(\omega)$ $\leqq \bar{x}(\omega)$ holds for each $\omega \in \Omega$. We will show that $\underline{x}=\bar{x}$ with probability 1. For each $\omega \in \Omega$ let $I(\omega)$ denote the union of the disjoint nontrivial closed intervals on which $F(\cdot, \omega)$ is constant. Since $\{\omega: \underline{x}(\omega)<\bar{x}(\omega)\}$ $C\{\omega: x(\omega) \in I(\omega)\}$, it will suffice to prove that $P(x(\omega) \in I(\omega))=0$. If Lemma 1 is applicable we have $P(x(\omega) \in I(\omega))=\int F(I(\omega), \omega) d P(\omega)=0$, under the assumption that the $\Omega \times \Lambda$ set $\Gamma$ whose $\omega$ sections are

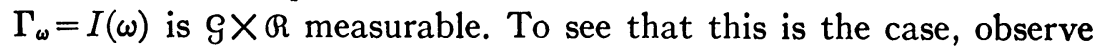
that $\Gamma=\lim _{n} \Gamma_{n}$, with

$$
\begin{gathered}
\Gamma_{n}=\underset{-\infty<j<k<\infty}{U} G_{n, j, k} \times\left[(j-1) / 2^{n},(k+1) / 2^{n}\right] \\
G_{n, j, k}=\left\{\omega: F\left((j-1) / 2^{n}, \omega\right)<F\left(j / 2^{n}, \omega\right)\right. \\
\left.=F\left(k / 2^{n}, \omega\right)<F\left((k+1) / 2^{n}, \omega\right)\right\} .
\end{gathered}
$$


Our main result is the following, to the effect that if there exists one such random variable $\xi$ then there exist many.

THEOREM 3. Suppose that there exists a random variable independent of $\mathcal{G}$ whose distribution is continuous. Then decomposition holds; that is, $\mathcal{G \vee G}=\mathcal{F}$

Proof. From Theorem 2, we may assume given a random variable $\xi$ with the property $P(\xi<u \mid \mathcal{G})=u$ with probability $1,0<u<1$. For arbitrary $A \in \mathcal{F}$ consider the random variable $\eta_{A}=\chi_{A}+\xi$, where $\chi_{A}$ denotes the characteristic function of set $A$. The conditional distribution of $\eta_{A}$ has the property

$$
\begin{aligned}
& P\left(\lambda_{1} \leqq \eta_{A}<\lambda_{2} \mid g\right)=P\left(A \cap\left\{\lambda_{1}-1 \leqq \xi<\lambda_{2}-1\right\} \mid \mathcal{G}\right) \\
& +P\left(A^{\prime} \cap\left\{\lambda_{1} \leqq \xi<\lambda_{2}\right\} \mid \mathcal{G}\right) \leqq 2\left(\lambda_{2}-\lambda_{1}\right)
\end{aligned}
$$

with probability 1 , implying that some version of $P\left(\eta_{A}<\lambda \mid \mathcal{G}\right)$ is continuous in $\lambda$ with probability 1 . Applying Theorem 2 to $\eta_{A}$, we obtain a random variable $\xi_{A}$ such that $\eta_{A}$ is $\left[\xi_{A}\right] \vee \mathcal{G}$ measurable and such that $\left[\xi_{A}\right] \subset \mathcal{G}^{s}$. Since $0<\xi<1$ with probability 1 , set $A$ differs from the set $\left\{\eta_{A}>1\right\}$ by a set of probability 0 , whence $A \in\left[\eta_{A}\right] \subset\left[\xi_{A}\right] \vee \mathcal{G} \subset \mathcal{S}^{8} \vee \mathcal{G}$ modulo set of probability 0 . But $A$ is an arbitrary element of $\mathcal{F}$, so that $\mathcal{F} \subset \mathcal{G} \vee \mathcal{S}^{s}$, or since the reverse inclusion is trivial, $\mathcal{F}=\mathcal{G} \vee \mathcal{S}^{s}$.

\section{Examples.}

Example 1. Decomposition holds. Let $(\Omega, \mathcal{F}, P)$ be atomless, and let $\mathcal{G}$ be purely atomic. That is, there is a finite or countable partition of $\Omega$ into disjoint sets $G_{i}, P\left(G_{i}\right)>0, i=1,2, \cdots$, and $\mathcal{G}$ is the $\sigma$-field generated by the $G_{i}, i=1,2, \cdots$. From Theorem $1, H \in \mathcal{G}^{*}$ if and only if $P\left(H \cap G_{i}\right) / P\left(G_{i}\right)$ is independent of $i$. To see that decomposition holds, let $A \in \mathcal{F}$ be an arbitrary measurable set, and define $H_{i i}=A \cap G_{i}, i=1,2, \cdots$. Since $(\Omega, \mathcal{F}, P)$ is atomless, for each $i$ there exist for all $j$ sets $H_{i j} \subset G_{j}$ such that $P\left(H_{i j}\right) / P\left(G_{j}\right)$ $=P\left(H_{i i}\right) / P\left(G_{i}\right)$. With $H_{i}=\cup_{j} H_{i j}$, we have $H_{i} \in \mathcal{G}^{s}, i=1,2, \cdots$, and finally $A=\bigcup_{i} H_{i i}=\bigcup_{i}\left(G_{i} \cap H_{i}\right) \in \mathcal{G} \vee \mathcal{G}^{8}$. We will prove also that $\mathcal{G}=\mathcal{G}^{s 8}$ modulo sets of probability 0 . Suppose $A \in \mathcal{F}$ does not differ from some $\mathcal{G}$ set by a set of probability 0 . Then both $P\left(A \cap G_{i}\right)>0$ and $P\left(A^{\prime} \cap G_{i}\right)>0$ hold for at least one $i$, say $i=j$. Choose $H_{j} \subset A \cap G_{j}$ and $K_{j} \subset A^{\prime} \cap G_{j}$ so that $P\left(H_{j}\right)=P\left(K_{j}\right)>0$. For all $k \neq j$ choose sets $H_{k}=K_{k} \subset G_{k}$ so that $P\left(H_{k}\right) / P\left(G_{k}\right)=P\left(H_{j}\right) / P\left(G_{j}\right)$. Defining $H=\bigcup_{l} H_{l}$, $K=\cup_{l} K_{l}$, we have $H, K \in \mathcal{G}^{s}$ and $P(H)=P(K)$. Since $P(A \cap H)$ $-P(A \cap K)=2 P\left(H_{j}\right)>0$, at least one of $H, K$ is not independent of $A$, implying $A \notin \mathcal{G}^{s s}$. Thus $\mathcal{G}^{s s} \subset \mathcal{G}$ modulo sets of probability 0 , whence $\mathcal{G}^{s}=\mathcal{G}$ modulo sets of probability 0 , from (1.vii). 
Example 2. Decomposition fails. Let $\Omega=\{(x, i): 0 \leqq x \leqq 1, i=1,2\}$, and let probability have density $p_{i}(x)$ at $(x, i)$ relative to linear Borel measure on the two intervals which comprise $\Omega$. Assume that $p_{1}(x)+p_{2}(x)=1,0 \leqq x \leqq 1$. If $B_{1}, B_{2}$ are Borel subsets of the unit interval we denote by $\left[B_{1}, B_{2}\right]$ the $\omega$ set $\left\{(x, 1): x \in B_{1}\right\}$ $\cup\left\{(x, 2): x \in B_{2}\right\}$. Let $\mathcal{G}$ be the sub- $\sigma$-field of sets of the form $[B, B]$ for all Borel $B$. It is readily verified that conditional probabilities relative to $\mathcal{G}$ have the form

$$
\begin{aligned}
& P\left(\left[B_{1}, B_{2}\right] \mid \mathcal{S}\right)_{(x, i)}=\chi_{B_{1}}(x) p_{1}(x)+\chi_{B_{2}}(x) p_{2}(x), \\
& 0 \leqq x \leqq 1, i=1,2 .
\end{aligned}
$$

For each $\omega$ the measure $P(\cdot \mid g)_{\omega}$ is purely atomic, the measures of the two atoms being $p_{1}(x), p_{2}(x)$. It is easy to choose $p_{1}, p_{2}$ in such a way that the only constant functions of the form (5) are 0 and 1 , which is to say, $g^{8}=0$. Thus decomposition fails. Note also that $\mathcal{G}^{8 s}=\mathcal{O}^{8}=\mathfrak{F} \neq \mathcal{G}$.

Example 2 suggests the following conjecture: for decomposition to hold it is sufficient that the conditional probability $P(\cdot \mid \xi)$ be an atomless measure with probability 1 . (This statement makes sense in the Stone representation space; see [3].)

5. Acknowledgment. Example 2 was suggested by Professor Murray Rosenblatt, and the author wishes to express his thanks to Professor Rosenblatt for a number of interesting conversations on this problem.

\section{REFERENCES}

1. J. L. Doob, Stochastic processes, John Wiley, New York, 1953.

2. P. Lévy, Theorie de l'addition variables aléatoires, Gauthier-Villars, Paris, 1937.

3. P. R. Halmos, On a theorem of Dieudonne, Proc. Nat. Acad. Sci. U.S.A. 35 (1949), 38-42.

Bell Telephone Laboratories, Murray Hill, New Jersey 